\title{
Genetically Encoded Immunophotosensitizer 4D5scFv-miniSOG is a Highly Selective Agent for Targeted Photokilling of Tumor Cells In Vitro
}

\author{
Kristina E. Mironova1 ${ }^{*}$, Galina M. Proshkina1 ${ }^{*}$, Anastasiya V. Ryabova ${ }^{2}$, Oleg A. Stremovskiy1, Sergey A. \\ Lukyanov $^{1,3}$, Rem V. Petrov ${ }^{1}$, and Sergey M. Deyev ${ }^{1,3}{ }^{凶}$ \\ 1. Shemyakin-Ovchinnikov Institute of Bioorganic Chemistry, Russian Academy of Sciences, Miklukho-Maklaya, 16/10, Moscow, 117997, \\ Russia; \\ 2. Prokhorov General Physics Institute, Russian Academy of Sciences, Vavilova, 38, 119991 Moscow, Russia; \\ 3. Research Institute of Applied and Fundamental Medicine, Nizhny Novgorod State Medical Academy, Nizhny Novgorod, Russia. \\ * These authors contributed equally to this work.
}

$\triangle$ Corresponding author: Sergey Deyev, Shemyakin-Ovchinnikov Institute of Bioorganic Chemistry, Russian Academy of Sciences, Miklukho-Maklaya, 16/10, Moscow, 117997, Russia. Phone 8(499)7247188; Fax: 8(499)7247188; E-mail: deyev@ibch.ru.

() Ivyspring International Publisher. This is an open-access article distributed under the terms of the Creative Commons License (http://creativecommons.org/ licenses/by-nc-nd/3.0/). Reproduction is permitted for personal, noncommercial use, provided that the article is in whole, unmodified, and properly cited.

Received: 2013.05.17; Accepted: 2013.08.09; Published: 2013.10.12

\begin{abstract}
Tumor-targeted delivery of cytotoxins presents considerable advantages over their passive transport. Chemical conjugation of cytotoxic module to antibody is limited due to insufficient reproducibility of synthesis, and recombinant immunotoxins are aimed to overcome this disadvantage. We obtained genetically encoded immunophotosensitizer 4D5scFv-miniSOG and evaluated its photocytotoxic effect in vitro. A single-chain variable fragment (scFv) of humanized 4D5 antibody was used as a targeting vehicle for selective recognition of the extracellular domain of human epidermal growth factor receptor 2 (HER2/neu) overexpressed in many human carcinomas. As a phototoxic module we used a recently described photoactivated fluorescent flavoprotein miniSOG. We found that recombinant protein $4 \mathrm{D} 5 \mathrm{scFv}$-miniSOG exerts a highly specific photo-induced cytotoxic effect on HER2/neu-positive human breast adenocarcinoma SK-BR-3 cells $\left(I C_{50}=160 \mathrm{nM}\right)$. We demonstrated that the $4 \mathrm{D} 5 \mathrm{scFv}$-miniSOG specifically binds to HER2-positive cells and internalizes via receptor-mediated endocytosis. Co-treatment of breast cancer cells with 4D5scFv-miniSOG and Taxol or junction opener protein JO-I produced remarkable additive effects.
\end{abstract}

Key words: tumor-targeted delivery, immunophototoxin, phototoxicity, HER2/neu, single-chain variable fragment.

\section{Introduction}

Photodynamic therapy (PDT) is a minimally invasive cancer therapy that includes accumulation of a photosensitizer in the tumor and subsequent illumination with light of a specific wavelength $[1,2]$.

Being in activated state, a photosensitizer (PS) transfers the energy from light to the molecular oxygen and generates reactive oxygen species (ROS, free radicals or singlet oxygen) that cause destruction of the targeted tissue. As ROS have short lifetime, the reactions of oxidative damage occur in the immediate vicinity of the PS. A short range of action $(\sim 10 \mathrm{~nm})$ in combination with precise light direction (for example, by means of fiber optics), ensures a high degree of spatial control during PDT. 
Chemically based photosensitizing reagents have been widely used in photodynamic therapy [1]. However, due to accumulation in normal tissue commercially available PS (Photofrin, Foscan and Verteporfin) cause side effects such as skin and eyes photosensitization.

To overcome this obstacle, a new approach for drug delivery, called targeted photodynamic therapy, has been developed $[3,4]$. One way for accurate delivery of PS to the tumor cells is chemical conjugation of PS to antibodies that recognize tumor-associated antigens. Following the pioneering work of Mew et al. [5] in the early 1980s the use of photosensitizer immunoconjugates became an advanced approach broadening the utility of photodynamic therapy [6]. Such macromolecular conjugates enable direct delivery of the PS to the target cell types. However, the main disadvantages of this approach are the limited reproducibility of the conjugate synthesis and the loss of activity of antibody or the PS [7, 8].

Another way for selective and effective therapeutic treatment is applying of genetically encoded PS.

We have previously reported a promising genetically encoded PS KillerRed, which can specifically recognize HER2/neu receptor (human epidermal growth factor receptor) and destroy adenocarcinoma cells in vitro due to KillerRed ability to generate ROS upon green light irradiation [9]. In general, HER2/neu, overexpressed on the surface of many cancer cells, is an important target for cancer therapy $[10,11]$. The first to demonstrate this in animals were Drebin and co-workers [12], who proved that monoclonal antibody specific to the extracellular domain of HER2/neu protein possesses a significant anti-tumor effect. This research became the keystone for development of an antibody-based therapy of cancer. 4D5 full-size humanized monoclonal antibody is widely used in clinical practice for immunotherapy of HER2/neu-overexpressing tumors [10, 13]. A recombinant $4 \mathrm{D} 5 \mathrm{scFv}$ mini-antibody is an example of high-efficiency HER2/neu-targeting vehicle that represents a single chain variable fragment of immunoglobulin (Ig) and exhibits lower cross-reactivity and immunogenicity and faster tissue penetration in comparison with the corresponding full-size antibody [13].

Recently a new highly phototoxic protein miniSOG (mini Singlet Oxygen Generator) has been reported [14]. This is a green fluorescent flavoprotein engineered from Arabidopsis phototropin 2, which uses flavin mononucleotide (FMN) as a cofactor. Upon blue-light illumination, miniSOG generates a sufficient quantity of singlet oxygen to induce dia- minobenzidine (DAB) photooxidation and also photoinduces cell ablation of neurons in C. elegans [15], making it a potentially useful tool for PDT and optogenetics [14-16].

We have taken into account the advantage of the ROS-producing properties of miniSOG and created the recombinant immunoPS consisting of an anti-HER2/neu 4D5 single chain variable fragment (4D5scFv) and miniSOG as a phototoxic module.

In this study we show that genetically encoded immuno-photosensitizer $4 \mathrm{D} 5 \mathrm{scFv}$-miniSOG specifically targets and photo-kills HER2/neu overexpressing cells but spare HER2-negative cells in vitro. Excellent $4 \mathrm{D} 5 \mathrm{scFv}$-miniSOG targeting and photokilling properties in vitro give its opportunity to be a reasonable immunoPS in vivo.

\section{Materials and Methods}

Construction of 4D5scFv-miniSOG expression plasmid. miniSOG coding region was amplified from pC1-miniSOG plasmid [17] using specific primers (5'GGTGGCGCGCCTATGGAAAAGAGCTTTGT, 5'GGCGGCGCGCCGCCATCCAGCTGCACACC).

The amplification product of $340 \mathrm{bp}$ was digested with AscI (recognition region is underlined in primer structure) and cloned under lac-promotor in one reading frame with the coding sequence of $4 \mathrm{D} 5 \mathrm{scFv}$ hybrid gene into pSD-4D5 [24]. E. coli colonies carrying inserts of miniSOG in direct orientation were selected. Generated plasmid pSD-4D5scFv-miniSOG was sequenced and proven to harbor correct coding sequence.

4D5scFv-miniSOG purification. Freshly transformed Escherichia coli SB536 strain [18] were grown in the YTPS medium ( $1 \%$ triptone, $1 \%$ yeast extract, 45 $\mathrm{mM} \mathrm{K} \mathrm{HPO}_{4}, 5 \mathrm{mM} \mathrm{KH} \mathrm{PO}_{4}, 0.1 \mathrm{M} \mathrm{NaCl}, 2 \mathrm{mM}$ $\mathrm{MgCl}_{2}$ ) containing $0.1 \mathrm{~g} / 1$ ampicillin; the lac-promoter was induced with $1 \mathrm{mM}$ IPTG at $\mathrm{OD}_{600}$ of 0.7. Expression was allowed to continue for $6 \mathrm{~h}$ at room temperature. Cells were harvested by centrifugation at $6,000 \mathrm{~g}$ for $10 \mathrm{~min}$ at $4^{\circ} \mathrm{C}$. Cell pellets were resuspended in lysis buffer $\left(200 \mathrm{mM} \mathrm{NaCl}, 30 \mathrm{mM} \mathrm{NaH} \mathrm{PO}_{4}, 2 \mathrm{mM}\right.$ Tris, $\mathrm{pH}$ 8.1) and then sonicated on ice in dark. Cellular debris was removed by centrifugation at $15,000 \mathrm{~g}$ and the supernatant was applied to a $\mathrm{Ni}^{2+}-\mathrm{NTA}$ column (GE Healthcare) according to the manufacturer's instruction. The immobilized proteins were eluted with $100 \mathrm{mM}$ imidazole and equilibrated on a PD10 column (GE Healthcare) in storage buffer (20 $\mathrm{mM} \mathrm{KH} \mathrm{KO}_{4}, 20 \mathrm{mM}$ HEPES, $10 \mathrm{mM}$ EDTA, $0.5 \mathrm{M}$ $\mathrm{NaCl}, 10 \%$ glycerol, $0.1 \%$ Tween, $\mathrm{pH} 8.0$ ). The yield of recombinant protein was $1.5 \mathrm{mg}$ purified protein per one liter of growth medium.

JO-1 purification. Plasmid pQE30-K-Ad3-6th- 
SK (provided by Andre Lieber) encoding JO-1 protein was expressed in XL1-Blue (Stratagene) and purified on $\mathrm{Ni}^{2+}-\mathrm{NTA}$ column (GE Healthcare) according to the manufacturer's instruction.

SDS-PAGE and western blot analysis. SDS-PAGE analysis was performed under denaturing conditions according to standard protocols using $12 \%$ polyacrylamide gels.

Band corresponding to $4 \mathrm{D} 5 \mathrm{scFv}$-miniSOG was excised from Coomasie blue-stained gel, digested with trypsin and subjected to MALDI-TOF mass spectrometry [19].

Immunoblots on Immobilon-P transfer membrane (Millipore) were carried out according to the manufacturer's instructions using anti-His tag mouse monoclonal antibody alkaline phosphotase conjugate (Abcam) for detection. The blots were visualized with nitroblue tetrazolium/5-bromo-4-chloro-3-indolyl phosphate.

Cell lines and incubation conditions. The human breast adenocarcinoma SK-BR-3 (HTB-30 ${ }^{\mathrm{TM}}$; ATCC) and Chinese hamster ovary $\mathrm{CHO}$ cell lines (Russian Cell Culture Collection) were maintained in RPMI-1640 medium (HyClone) supplemented with $10 \%$ fetal calf serum (HyClone) and $2 \mathrm{mM}$ L-glutamine (PanEko). Cells were incubated in a 5\% $\mathrm{CO}_{2}$ atmosphere at $37^{\circ} \mathrm{C}$.

Internalization/plasma membrane localization stadies. SK-BR-3 cells were cultured on chamber-slides (Nalge Nunc International) overnight at $37^{\circ} \mathrm{C}$, washed and incubated with $500 \mathrm{nM}$ $4 \mathrm{D} 5 \mathrm{scFv}$-miniSOG at $4^{\circ} \mathrm{C}$ for $1 \mathrm{~h}$ following plasma membrane localization studies. For 4D5scFv-miniSOG internalization, cells were further washed and incubated for additional $30 \mathrm{~min}$ at $37^{\circ} \mathrm{C}$. CHO cells were used as nontarget cells (negative control) to demonstrate HER2/neu-specific binding of $4 \mathrm{D} 5 \mathrm{scFv}$-miniSOG. The slides were analyzed with the laser scanning microscope Carl Zeiss LSM-710-NLO (Prokhorov General Physics Institute, Russian Academy of Sciences).

Competition experiments. For receptor blocking studies SK-BR-3 cells were seeded in chamber-slides (Nalge Nunc International) at the density of 30,000 cells/ml in RPMI-1640 medium and grown overnight at $37^{\circ} \mathrm{C}$. After that the conditioned medium was replaced with the medium containing $500 \mathrm{nM}$ of $4 \mathrm{D} 5 \mathrm{scF}$-miniSOG and incubated for $1 \mathrm{~h}$ at $37^{\circ} \mathrm{C}$. Competitive assay was performed in the presence of equimolar concentration of $4 \mathrm{D} 5 \mathrm{scFv}$. Fluorescence intensity of treated cells was examined using laser scanning microscope Carl Zeiss LSM-710-NLO with excitation at $458 \mathrm{~nm}$.

Subcellular localization studies. SK-BR-3 cells were cultured on chamber-slides (Nalge Nunc International) overnight at $37^{\circ} \mathrm{C}$, washed, treated with 500 $\mathrm{nM} 4 \mathrm{D} 5 \mathrm{scF}$-miniSOG at $4^{\circ} \mathrm{C}$ for $1 \mathrm{~h}$ and washed twice with cold PBS again. Than the cells were stained with specific dyes for mitochondria or for lysosome (MitoTracker Red and LysoTracker Red, Invitrogen) for $20 \mathrm{~min}$ and $3 \mathrm{~h}$ respectively at $37^{\circ} \mathrm{C}$ according to the manufacturer's instruction. After that cells were washed with PBS for 3 times and observed under the laser scanning microscope Carl Zeiss LSM-710-NLO (Prokhorov General Physics Institute, Russian Academy of Sciences). The $4 \mathrm{D} 5 \mathrm{scFv}$-miniSOG was excited at $458 \mathrm{~nm}$. The MitoTracker Red and LysoTracker Red organelle probe dyes were excited at the $561 \mathrm{~nm}$. Colocalization analysis of photomicrographs was performed using the Carl Zeiss LSM-710-NLO software.

Cell viability assay (MTT). The effect of $4 \mathrm{D} 5 \mathrm{scF}$-miniSOG or miniSOG on cell viability was studied using the microculture tetrazoline test (MTT) [20]. Briefly, SK-BR-3 and CHO cells were seeded in 96-well microtiter plates at 25,000 and 30,000 cells/ml, respectively. After $24 \mathrm{~h}$ media was removed, and the cells were treated with $4 \mathrm{D} 5 \mathrm{scFv}$-miniSOG or miniSOG at concentrations ranging from $1 \mathrm{nM}$ to $500 \mathrm{nM}$. To investigate $4 \mathrm{D} 5 \mathrm{scFv}$-miniSOG cytotoxic action in combination with Taxol (Paclitaxel) or junction opener protein JO-1, cells were treated with 4D5scFv-miniSOG ( 1 to $500 \mathrm{nM}$ ) in the presence of 1 $\mu \mathrm{M}$ Taxol or $5 \mu \mathrm{g} / \mathrm{ml}$ JO- 1 . To avoid unspecific effects the storage buffer was replaced with the RPMI-1640 medium. After $1 \mathrm{~h}$ incubation at $37^{\circ} \mathrm{C}$ the media was replaced by a fresh portion and the cells were exposed to white light with $1 \mathrm{~W} / \mathrm{cm}^{2}$ from a lamp Dolan-Jenner Fiber-Lite 180 Illuminator $\left(1 \mathrm{~W} / \mathrm{cm}^{2}\right.$ of white light gives $55 \mathrm{~mW} / \mathrm{cm}^{2}$ of blue light within the efficient absorption range of $4 \mathrm{D} 5 \mathrm{scF}$-miniSOG) $10 \mathrm{~min}$. After $48 \mathrm{~h}$ incubation, growth medium was replaced by RPMI-1640 containing $0.5 \quad \mathrm{mg} / \mathrm{mL}$ 3-(4,5-dimethylthiazol-2-yl)-2,5-diphenyltetrazolium bromide. Cells were incubated at $37^{\circ} \mathrm{C}$ for $2 \mathrm{~h}$, then the medium was withdrawn and $100 \mu \mathrm{l}$ of DMSO (Sigma) was added to each well. Cell viability was quantified spectrophotometrically at $540 \mathrm{~nm}$ using a microplate reader (Awareness Technology, INC. USA). The cell viability was calculated as the percentage of proliferating cells relative to the number of untreated cells. All experiments were performed in triplicate.

Statistical analysis. All results are expressed as means \pm standard deviations (SD). The statistical differences between groups of data were analyzed by one-way ANOVA. P $<0.05$ was considered significant. 
Absorbance and fluorescence spectroscopy. Absorbance spectra (400-600 nm) of purified proteins $(10 \mu \mathrm{M})$ were recorded on a Hitachi U-3400 spectrophotomer at $25^{\circ} \mathrm{C}$. Emission spectra were recorded on a laser scanning microscope Carl Zeiss LSM-710-NLO in $\lambda$-mode regime at the excitation wavelength of 458 $\mathrm{nm}$.

$K_{\mathrm{D}}$-Determination by surface plasmon resonance. Dissociation constant $\left(K_{D}\right)$ was determined by surface plasmon resonance with a BIAcore instrument (GE Healthcare Life Sciences). CM-5 sensor chips were coated with 1500 or 5000 resonance units of p185 ${ }^{\mathrm{HER}-2-\mathrm{ECD}}$ antigen. $4 \mathrm{D} 5 \mathrm{scFv}$-miniSOG in concentration of $5 \mu \mathrm{M}$ was injected in a final volume of $30 \mu \mathrm{l}$ on the chips. Dissociation rate constant was calculated from the sensorgram using the BIAevaluation 3.0 software (GE Healthcare Life Sciences).

\section{Results}

\section{Design and characteristics of 4D5scFv- miniSOG}

It has been previously shown that natural $\mathrm{N}$-terminus of $4 \mathrm{D} 5 \mathrm{scFv}$ is important for proper antibody function [21]. Thus, the nucleotide sequence of the hybrid gene encoding anti-HER2/neu 4D5scFv fragment was placed at the 5 -terminus of the miniSOG coding sequence. The target and phototoxic domains were connected via flexible linker that prevented spatial interference between the two domains (Fig. 1A).

The resulting genetic construct (Fig. 1B) was expressed in E. coli SB536 strain [18] as the C-terminus $\mathrm{His}_{5}$-tag protein and purified using $\mathrm{Ni}^{2+}$-NTA affinity chromatography (Fig. 2A).

As was determined by SDS-PAGE electrophoresis, the produced $4 \mathrm{D} 5 \mathrm{scFv}$-miniSOG had an expected molecular mass ( $\left.44 \mathrm{kDa}, \mathrm{Mw}_{\text {theor }} 42 \mathrm{kDa}\right)$ and was homogenous. The identity of the purified protein was verified by western blot analysis (Fig. 2B) and MALDI-TOF mass-spectrometry.

Absorption and emission spectra of 4D5scFv-miniSOG and free miniSOG are demonstrated in Fig. 2C. Excitation of the hybrid protein $4 \mathrm{D} 5 \mathrm{scFv}$-miniSOG leads to green emission with the two peaks at 500 and $526 \mathrm{~nm}$ (Fig. 2C) that is in good agreement with the fluorescence spectra for free miniSOG [14].

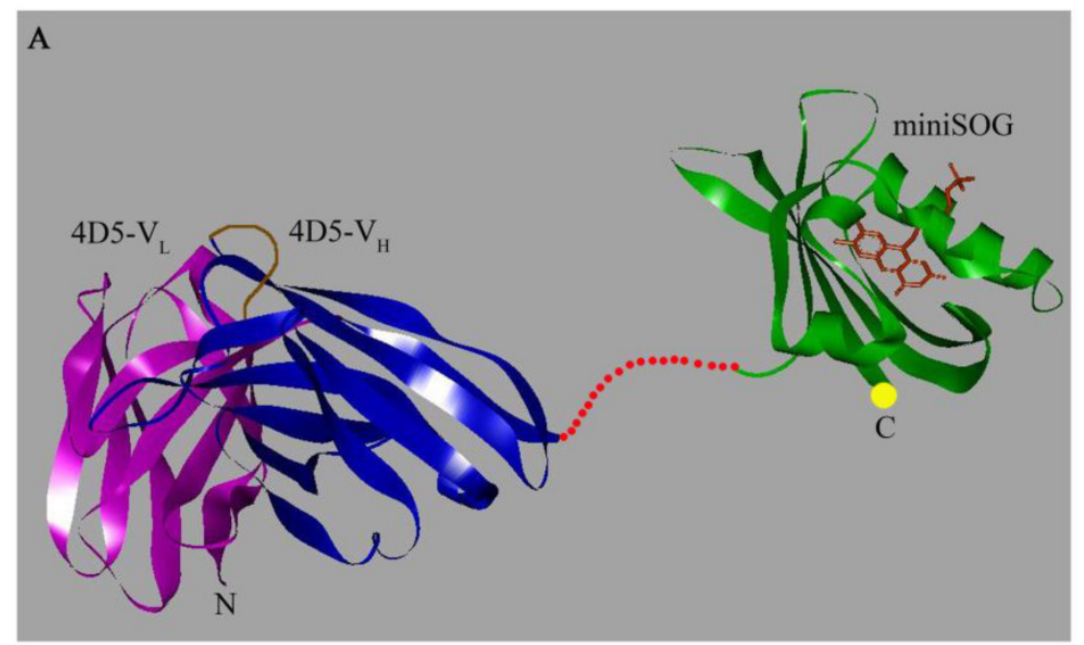

B

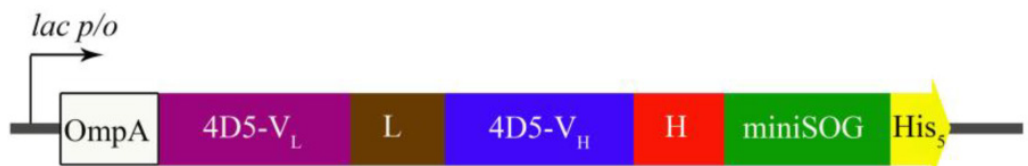

Fig I. 3D model and gene construct of the genetically encoded immunoPS. A, Molecular model (ribbon representation) of the 4D5scFv (PDB entry IFCV) [22] and miniSOG. The 3D structure of miniSOG was made using SWISS-MODEL structure homology-modeling server [23, 24]. Cofactor FMN is shown in the miniSOG chromophore pocket. The image of 3D immunoPS structure was made using DS ViewerPro 5.0 software. B, Gene construct for expression of the 4D5scFv-miniSOG. OmpA, signal peptide for direct secretion of the recombinant protein to the periplasmic space of $E$. coli (white); $V_{L}$, light chain of 4D5scFv (magenta); $L$, designed peptide linker (2l a.a., brown) that links the carboxyl terminus of the $V_{L}$ sequence to the amino terminus of the $V_{H}$ sequence; $\mathrm{V}_{\mathrm{H}}$, heavy chain (blue); $\mathrm{H}$, hinge-like linker ( 16 a.a., red) derived from the murine lgG3 hinge region; miniSOG, coding region of miniSOG (green); $\mathrm{His}_{5}$, C-terminal 5-His-tag (yellow). 
A

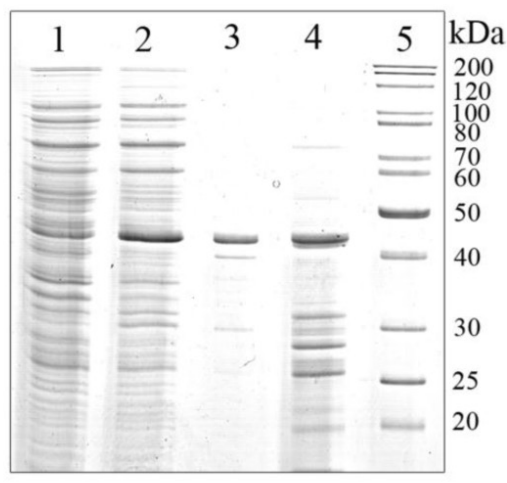

$\mathrm{C}$

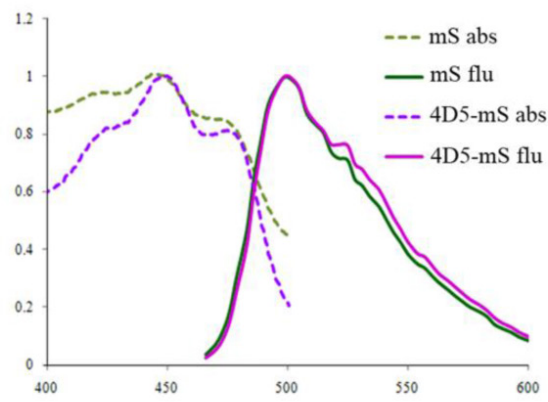

B

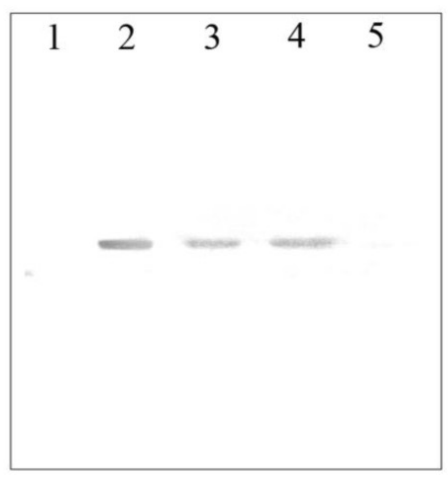

$\mathrm{D}$

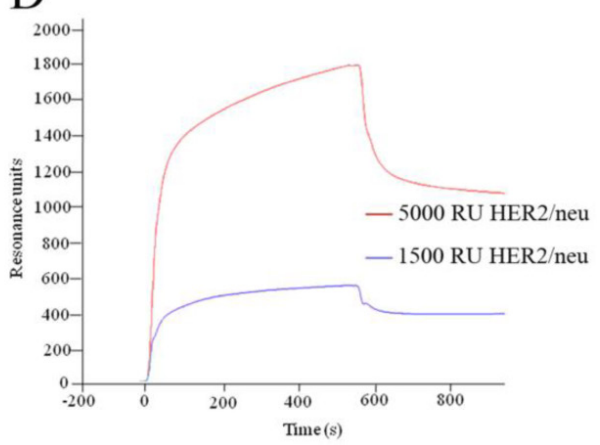

Fig 2. $4 \mathrm{D} 5 \mathrm{scFv}$-miniSOG purification and characteristics. A, SDS-PAGE analysis of the purified recombinant 4D5scFv-miniSOG. Coomassie blue-stained polyacrylamide gel: uninduced cell extract of $E$. coli strain SB536 (Lane I); whole lysate of the same bacterial cells after induction with IPTG (Lane 2); purified 4D5scFv-miniSOG (Lane 3); insoluble fraction (Lane 4); molecular weight markers of proteins PageRuler Unstained Protein Ladder, Fermentas (Lane 5). B, Western blot analysis of immunoPS with antibodies directed against the His -tag $_{5}$ (Lanes I-5 correspond to Lanes I- 5 in A). C, Normalized absorbance (dotted light-green line for free miniSOG $(\mathrm{mS})$ and dotted lilac line for 4D5scFv-miniSOG (4D5-mS)) and emission spectra (green line for free miniSOG and pink line for 4D5scFv-miniSOG) of the proteins; D, SPR sensorgram of 4D5scFv-miniSOG interaction with CM-5 sensor chips. Violet line represents the binding of 4D5scFv-miniSOG with CM-5 sensor chips coated with recombinant HER2/neu-receptor at density of I 500 RU, red line - at density of 5000 RU.

\section{Evaluation of the HER2/neu binding}

Binding of $4 \mathrm{D} 5 \mathrm{scFv}$-miniSOG to a purified HER2/neu receptor was evaluated by Plasmon surface resonance (Fig. 2D). The $K_{D}$ value of the $4 \mathrm{D} 5 \mathrm{scFv}$-miniSOG to HER2/neu receptor was determined by BIAcore to be $10 \pm 2 \mathrm{nM}$, which corresponds well to that of $4 \mathrm{D} 5 \mathrm{scFv}(9 \pm 2 \mathrm{nM})$ [25].

Breast adenocarcinoma SK-BR-3 cells, which express $\sim 1 \times 10^{6}$ to $2 \times 10^{6} \mathrm{HER} 2 /$ neu receptors per cell [26], were chosen as a model cell line. As was determined by confocal microscopy, 4D5scFv-miniSOG accumulated effectively on the cellular membrane surface after $1 \mathrm{~h}$ incubation at $4^{\circ} \mathrm{C}$ (Fig. 3A). After removing all non-specifically bounded $4 \mathrm{D} 5 \mathrm{scFv}$ miniSOG by washing with PBS, the cells were left for $30 \mathrm{~min}$ at $37^{\circ} \mathrm{C}$ for internalization. Fluorescent signal inside the SK-BR-3 cells on the micrograph (Fig. 3B) clearly indicates the ability of $4 \mathrm{D} 5 \mathrm{scFv}$-miniSOG to penetrate into the cells by receptor-mediated endocytosis. Fluorescent signal was undetectable when free miniSOG was added to SK-BR-3 cells (Supple- mentary Material: Fig. S1A). No binding activity was detected in the case of $\mathrm{CHO}$ cells (negative control) that do not overexpress HER2/neu (Supplementary Material: Fig. S1B).

\section{Competition experiments}

$4 \mathrm{D} 5 \mathrm{scF}$-miniSOG binding specific properties was also investigated in competition experiments with $4 \mathrm{D} 5 \mathrm{scFv}$ mini-antibody. Figure 4 clearly demonstrates that SK-BR-3 binding of $4 \mathrm{D} 5 \mathrm{scFv}-\mathrm{miniSOG}$ was inhibited when competed with $4 \mathrm{D} 5 \mathrm{scFv}$. Integrated fluorescence intensity signal for SK-BR-3 cells treated with $500 \mathrm{nM} 4 \mathrm{D} 5 \mathrm{scF}$-miniSOG was in 2.5-fold greater than for SK-BR-3 cells treated with 500 $\mathrm{nM} 4 \mathrm{D} 5 \mathrm{scFv}$-miniSOG in the presence of $500 \mathrm{nM}$ 4D5scFv (Fig. 4C). The SK-BR-3 cell amount was equal in both cases.

\section{Intracellular localization}

For a better understanding of the immunoPS-mediated PDT, we characterized the intracellular localization of the immunoPS and quantified 
the colocalization patterns with lysosomes and mitochondria.

Micrographs of the SKBR-3 cells double stained with 4D5scFv-miniSOG and LysoTracker Red or MitoTrackerRed are presented in Figure 5 . $4 \mathrm{D} 5 \mathrm{scFv}$-miniSOG was shown to localize mainly in the cytoplasm. Only weak fluorescence intensity was found in the nucleus. Yellow areas correspond to the colocalization fraction of $4 \mathrm{D} 5 \mathrm{scFv}$-miniSOG and organelle specific dyes (Figures 5Aiii and 5Biii). Thus, lysosomes localized mainly at the same site of the cytoplasm where 4D5scFv-miniSOG accumulation occurred (Figure 5Aiii). But yellow areas were slightly detectable in cells double-stained with $4 \mathrm{D} 5 \mathrm{scFv}-$ miniSOG and the MitoTrackerRed (Figure 5Biii).

In order to increase the significance of the co-staining experiments, fluorescence images were analyzed quantitatively by the Carl Zeiss LSM-710-NLO software, and results are depicted in scatterplots (Figure 5Aiv, Biv).

Colocalization coefficients were calculated as relative number of colocalizing pixels in channel 1 or
2, respectively, as compared to the total number of pixels above threshold. In cells double stained with $4 \mathrm{D} 5 \mathrm{scF}$-miniSOG and LysoTracker, the colocalization fraction of $4 \mathrm{D} 5 \mathrm{scFv}$-miniSOG found in the lysosomes was $21 \%$ (Ch2-T1), whereas $72 \%$ (Ch1-T1) of the lysosome fluorescence coincided with the 4D5scFv-miniSOG fluorescence (Figure 5Aiv). Due to receptor-mediated nature of $4 \mathrm{D} 5 \mathrm{scFv}$-miniSOG endocytosis it was of our expectation to observe its localization in lysosomes. In the case of cells double stained with $4 \mathrm{D} 5 \mathrm{scF}$-miniSOG and MitoTracker, there was also some fluorescence coincidence in mitochondrions vs $4 \mathrm{D} 5 \mathrm{scF}$-miniSOG and vice versa (colocalisation coefficients were $28 \%$ (Ch2-T1) and 23\% (Ch1-T2) respectively) (Figure 5Biv).

Thus, the majority of the immunoPS is located in cytoplasm. As we previously proved an absence of unspecific entry of $4 \mathrm{D} 5 \mathrm{scFv}$-miniSOG through the cell membrane it may indicate its endosomal localization or the photo-induced release of $4 \mathrm{D} 5 \mathrm{scFv}$-miniSOG from lysosomes under laser scanning manipulation.
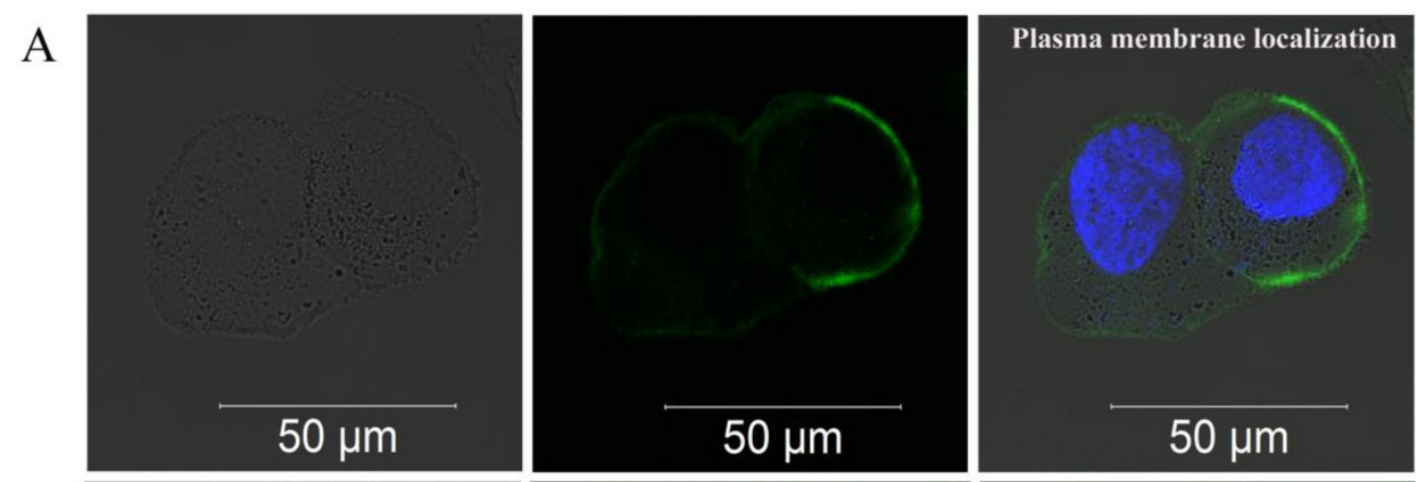

B
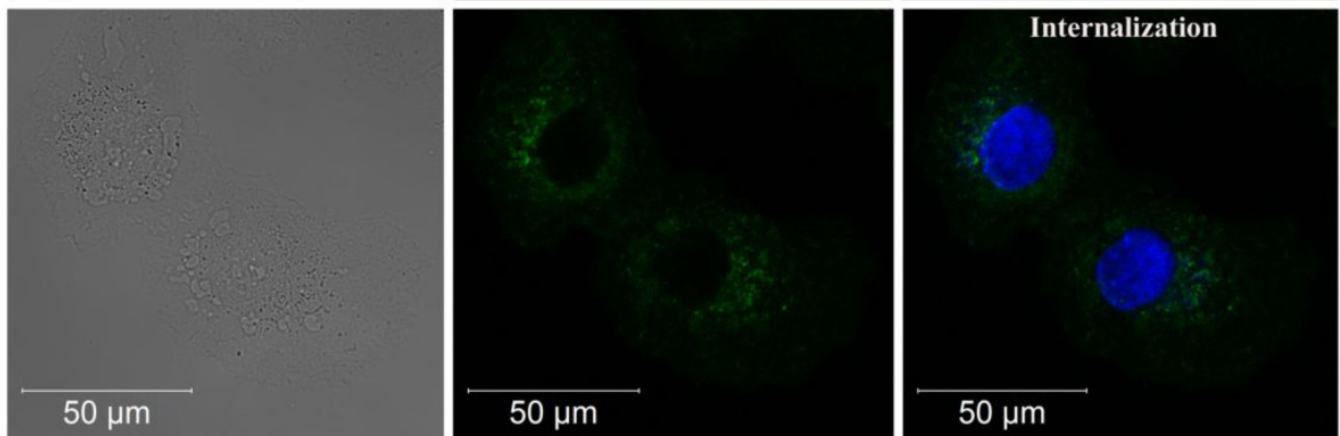

Fig 3. Confocal fluorescence images of SK-BR-3 cells after I $\mathrm{h}$ incubation with $4 \mathrm{D} 5 \mathrm{scFv}$-miniSOG at $4^{\circ} \mathrm{C}(\mathrm{A})$ and after additional 30 min incubation at $37^{\circ} \mathrm{C}$ (B). Left row, a bright-field image; middle row, fluorescence image in green channel; right row, fluorescence image in green and blue channels (nuclei stains with Hoechst 33258). 

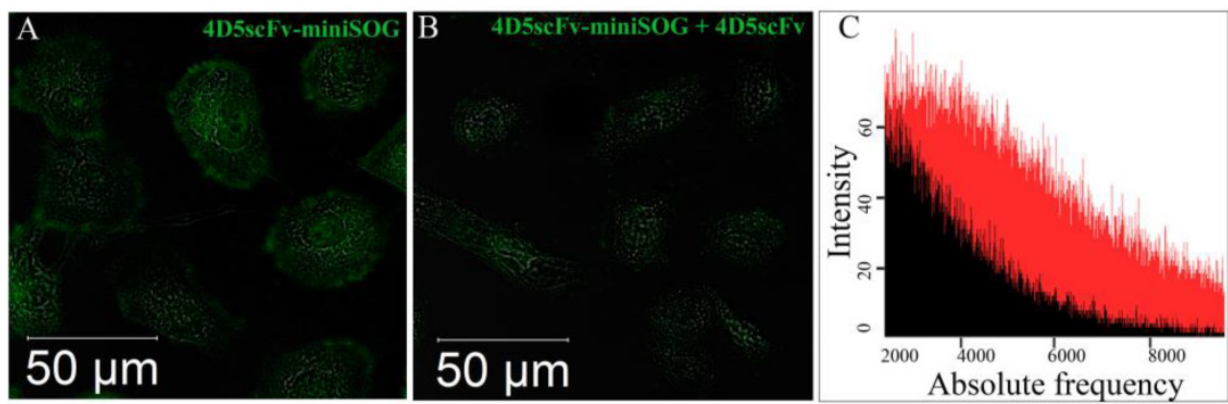

Fig 4. 4D5scFv-miniSOG competitive uptake experiments. HER2/neu-overexpressing SK-BR-3 cells were incubated for $1 \mathrm{~h}$ at $37^{\circ} \mathrm{C}$ with $500 \mathrm{nM}$ 4D5scFv-miniSOG (A) or $500 \mathrm{nM} 4 \mathrm{D} 5 \mathrm{scFv}$-miniSOG plus $500 \mathrm{nM}$ 4D5scFv (competitive mini-antibody) (B). The integral signal of fluorescence in both cases were measured using confocal microscopy and presented as a histogram (C): red field -fluorescence signal corresponding to 4D5scFv-miniSOG-treated cells, black field - fluorescence signal corresponding to 4D5scFv-miniSOG plus 4D5scFv-treated cells.
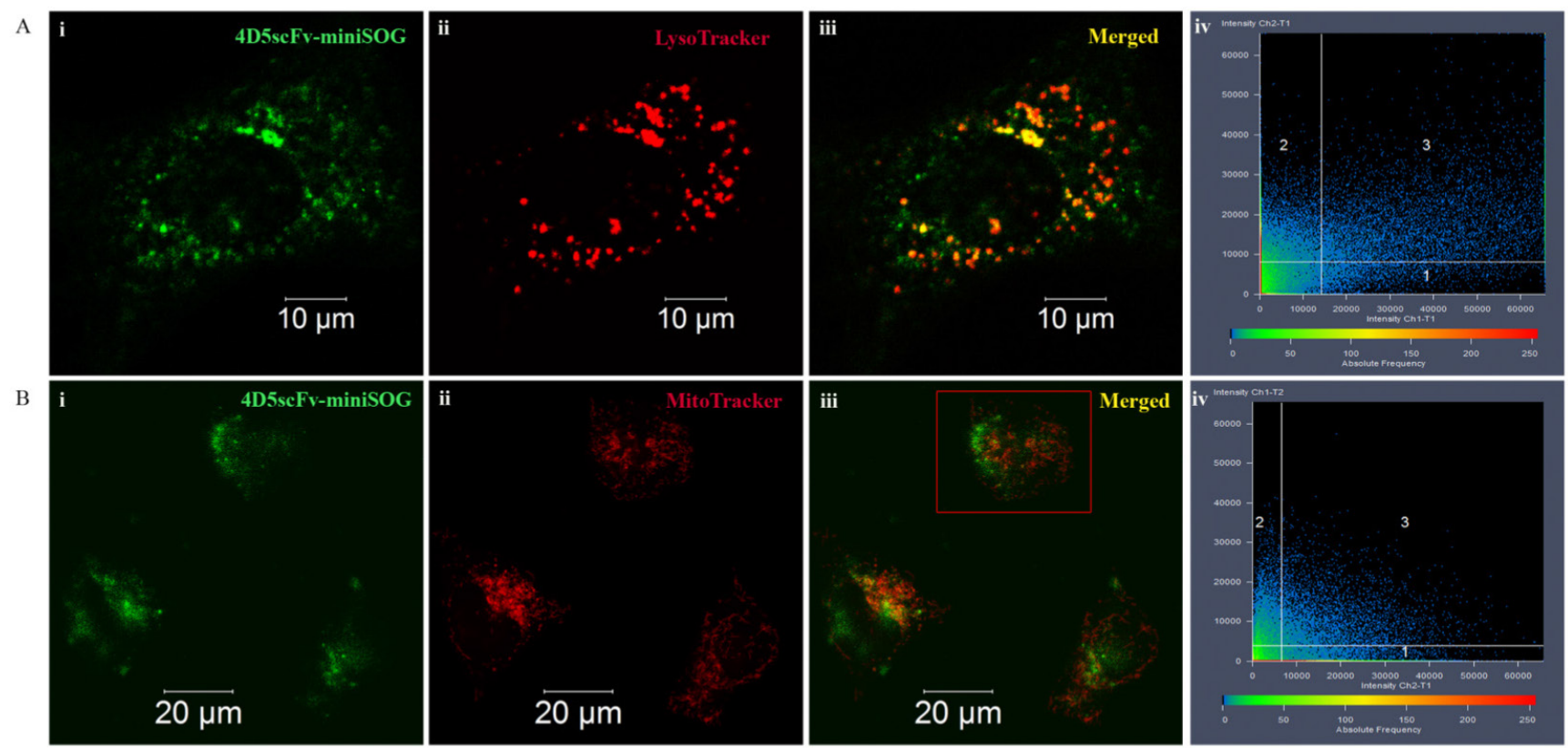

Fig 5. 4D5scFv-miniSOG localization in different organelles in SK-BR-3 cells. 4D5scFv-miniSOG vs LysoTracker (A), 4D5scFv-miniSOG vs MitoTracker (B). Representative 4D5scFv-miniSOG images of the cells are shown in green (i), images of each organelle-specific dye are shown in red (ii), and merged fluorescent images are displayed in iii. Scatterplots of $4 \mathrm{D} 5 \mathrm{scFv}$-miniSOG and organelle-specific dye signal frequency and intensity are shown in iv. The cell on fig. Biii indicated in red frame was chosen for scatterplot measurement.

\section{Specific photo-induced cytotoxicity of 4D5scFv-miniSOG}

In order to estimate the specific photo-induced cytotoxic effect of $4 \mathrm{D} 5 \mathrm{scFv}-\mathrm{miniSOG}$ on the viability of SK-BR-3 cells, a microculture tetrazolium test (MTT) was used. Decreasing concentrations of immunoPS were added to SK-BR-3 cells, than incubated for $1 \mathrm{~h}$ at $37^{\circ} \mathrm{C}$, followed by exposure to light from a lamp (Dolan-Jenner Fiber-Lite 180 Illuminator) with 1 $\mathrm{W} / \mathrm{cm}^{2}$ of bright white light $\left(55 \mathrm{~mW} / \mathrm{cm}^{2}\right.$ of blue light within the efficient absorption range of $4 \mathrm{D} 5 \mathrm{scFv}$-miniSOG) for $10 \mathrm{~min}$, the percentage of viable cells was determined compared to the untreated cells. Using regression analysis, $\mathrm{IC}_{50}$ was estimated as $160 \mathrm{nM}$. We observed that the maximal cytotoxic ef- fect $(21 \%$ of viable cells) for SK-BR-3 cells was achieved at $500 \mathrm{nM}$ of $4 \mathrm{D} 5 \mathrm{scFv}$-miniSOG (Fig. 6A). It should be noted that this cytotoxic effect of $4 \mathrm{D} 5 \mathrm{scFv}$-miniSOG was observed under $55 \mathrm{~mW} / \mathrm{cm}^{2}$ blue light intensity, but not under typical indoor illumination $\left(\sim 0.2 \mathrm{~mW} / \mathrm{cm}^{2}\right)$ (Fig. $\left.6 \mathrm{~A}\right)$. ImmunoPS did not affect viability of the $\mathrm{CHO}$ cells in the dark or under white light even if treated with maximum concentration of immunoPS (Fig. 6B).

Free $4 \mathrm{D} 5 \mathrm{scFv}$, free miniSOG and free FMN did not affect cell viability (Fig. 6C), indicating high specificity of the 4D5scFv-miniSOG phototoxic effect.

We also investigated the cytotoxic action of 4D5scFv-miniSOG in combination with mitotic inhibitor Taxol (Paclitaxel) or junction opener protein JO-1 [27]. 
Taxol is a well-known cytostatic drug, and in our experiment it was used to estimate the cytostatic action of $4 \mathrm{D} 5 \mathrm{scFv}$-miniSOG in non-proliferating cell culture. Checking several toxic concentrations of Taxol we have found that $1 \mu \mathrm{M}$ of anticancer drug causes $40 \%$ cell death (Supplementary Material: Fig. S2), which is in good agreement with literature data [28]. This concentration of Taxol was further used in toxicity experiments. SK-BR-3 cells were treated with various concentrations of $4 \mathrm{D} 5 \mathrm{scFv}$-miniSOG and 1 $\mu \mathrm{M}$ of Taxol, and the percentage of viable cells relative to the immunoPS/drug-free control was determined. We have found that $1 \mu \mathrm{M}$ of Taxol significantly increased citotoxicity of $4 \mathrm{D} 5 \mathrm{scFv}$-miniSOG, so that co-treatment produced $100 \%$ cell death (Fig. 6A). On the other hand, the same concentration of Taxol
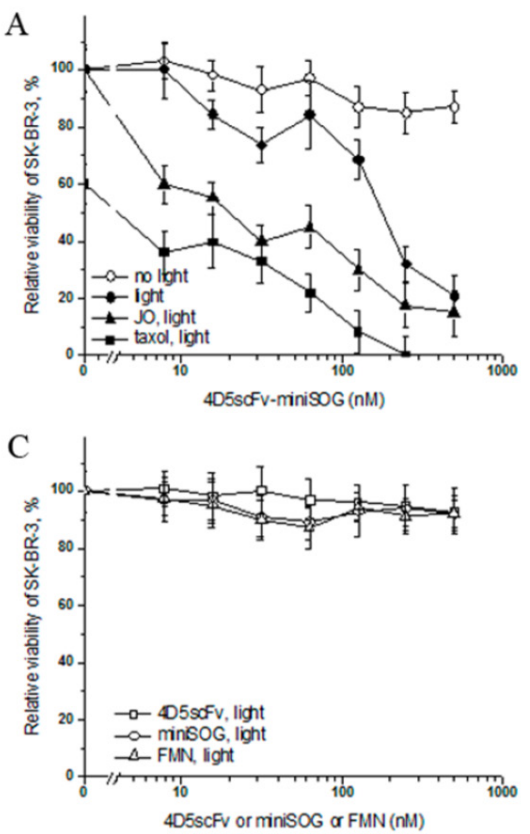

showed no increase in $4 \mathrm{D} 5 \mathrm{scF}$-miniSOG citotoxicity on $\mathrm{CHO}$ cells (Fig. 6B).

Another agent that we used in our experiments was junction opener protein JO-1. JO-1 is a self-dimerizing recombinant protein derived from the adenovirus serotype 3 fiber [27]. The pretreatment of epithelial cells with JO-1 resulted in increased access to receptors that are localized in or masked by epithelial junctions [27]. JO-1 was shown recently to increase intratumoral penetration of the anti-HER2/neu $\mathrm{mAb}$ Herceptin [29]. We tested weather JO-1 would improve SK-BR-3 killing by immunoPS. Cells in 100\% confluency were co-incubated with JO-1 and $4 \mathrm{D} 5 \mathrm{scFv}$-miniSOG for $2 \mathrm{~h}$ and then irradiated with white light. Addition of $5 \mu \mathrm{g} / \mathrm{ml} \mathrm{JO}-1$ decreased $\mathrm{IC}_{50}$ of $4 \mathrm{D} 5 \mathrm{scFv}$-miniSOG from 160 to $5 \mathrm{nM}$ (Fig. 6A).

B

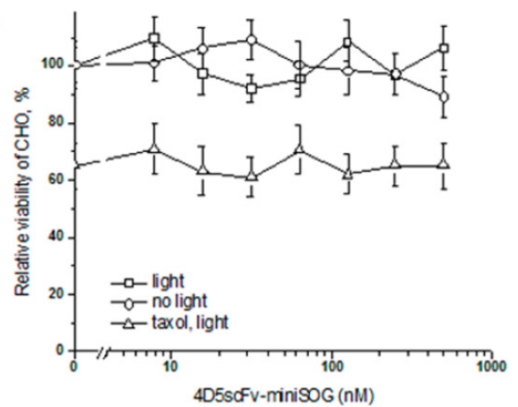

Fig 6. In vitro cytotoxicity analysis of $4 \mathrm{D} 5 \mathrm{scFv}$-miniSOG. A, The impact of $4 \mathrm{D} 5 \mathrm{scFv}$-miniSOG decreasing concentrations on the SK-BR-3 cell viability under different conditions: white light and dark, taxol or JO-I cotreatment. B, Relative viability of $\mathrm{CHO}$ after the treatment with 4D5scFv-miniSOG under white light or in the dark and after the co-treatment with 4D5scFv-miniSOG and Taxol under white light. C, Effects of free 4D5scFv, free miniSOG and free FMN on the SK-BR-3 cell viability. Bars indicate SD for 3 independent experiments. $P<0.05$.

\section{Discussion}

In this study we have clearly demonstrated that genetically encoded PS 4D5scFv-miniSOG can be successfully used for tumor cell destruction in vitro.

Our experiments indicate that photo-damage of tumor cells by targeting the $4 \mathrm{D} 5 \mathrm{scFv}$-miniSOG to HER2/neu-overexpressing cells is highly specific only for these cells and occurs only upon light irradiation. Being in fuse with miniSOG, $4 \mathrm{D} 5 \mathrm{scFv}$ reserves binding activity to HER2/neu $\left(K_{D}=10 \pm 2 \mathrm{nM}\right)$ and at the same time miniSOG retains its ability to generate ROS, which is proved by its cytotoxic effect on
SK-BR-3 cells in vitro. Our data (Figs. 4 and 6) clearly show that the phototoxic effects of $4 \mathrm{D} 5 \mathrm{scFv}$-miniSOG is mediated via specific HER2/neu targeting and is not due to the nonspecific effects.

In vitro cell killing using $4 \mathrm{D} 5 \mathrm{scFv}$-miniSOG is much effective than in the case of $4 \mathrm{D} 5 \mathrm{scFv}-K$ KillerRed [9]. Being compared to anti-HER2/neu scFv-photosensitizer immunoconjugates, 4D5scFvminiSOG showed a greater than 8 -fold and 30 -fold improvement of $\mathrm{IC}_{50}$ over anti-HER2/neupyropheophorbide-a and anti-HER2/neu-verteporfin respectively [30]. Most of photosensitizers used in photodynamic therapy are hydrophobic and lipo- 
philic and tend to aggregate in aqueous solutions. So it is not easy to conjugate them to antibodies. Besides this many photosensitizer immunoconjugates can contain some amounts of free photosensitizer impurities. Also conjugation can sterically hinder or disrupt antibody antigen binding sites [31]. Being genetically-encoded, 4D5scFv-miniSOG has fixed photosensitizer/antibody ratio, a defined junction of two functional modules, absence PS impurity, as well as stable and simple production of the active recombinant protein in bacteria.

There is one more approach utilizing miniSOG as a genetically encoded photosensitizer for cancer cells has been recently published [17]. Ryumina and co-workers received HeLa Kyoto cell lines stably expressing miniSOG and established its phototoxic effect in vitro. But they did not observe photoinduced tumor reduction in vivo. Authors believe that such unexpected in vivo result can be due to unsaturation of miniSOG by its cofactor FMN. In this case our approach based on the production of miniSOG in bacteria has one crucial advantage: bacterial expression allows to obtain miniSOG fully saturated with FMN which is extremely necessary for strong damage of cancer cells. Moreover we produce HER2/neutargeting miniSOG that broadens the utility of PS in vivo.

Generally, photosensitizers can generate type I (photoinduced electron transfer) or type II (energy transfer from exited photosensitizer to oxygen) photochemical reactions. It has been previously shown that due to bounded FMN, excited miniSOG generates singlet oxygen with a high yield (0.47) [14] and thus to be a type II photosensitizer. In contrast to this claim there are two recent publications stated that miniSOG indeed is a very poor singlet oxygen sensitizer, and FMN being enclosed in miniSOG produces a reactive species other than ${ }^{1} \mathrm{O}_{2}[32,33]$. So miniSOG may be considered as type I photosensitizer. Taking into account all the statements $[32,33]$ and our in vitro results we can conclude that miniSOG does initiate photochemical reactions defining its high cytotoxic properties. This result can be due to both type I and type II photochemical reactions.

Combining PDT with other therapies is known to increase effectiveness of anti-tumor treatments [1, 34]. We showed that co-treatment of cells with immunoPS and mitotic inhibitor Taxol leads to $100 \%$ SK-BR-3 cells death, while complete cell killing was not achieved if $4 \mathrm{D} 5 \mathrm{scF}$-miniSOG was applied alone (Fig. 6A). This strategy gives opportunity to use drugs in lower concentrations and decreases possibility of side effects.

Another way for the reduction of immunoPS doses is to improve an access to its target receptor. It has been shown previously, that adenovirus junction opener protein JO-1 is capable of transient opening of intercellular junctions, leading to increase of intratumoral penetration of the anti-HER2/neu monoclonal antibody trastuzumab (Herceptin) [29]. We have found that JO-1 enhances immunoPS cytotoxicity, significantly improving the outcome of the breast cancer treatment with 4D5scFv-miniSOG (Fig. 6A).

It is obvious that the efficacy of the diseased tissue destruction during photodynamic therapy depends on the depth of the light penetration. Blue light has only limited propagation through the tissues. As 4D5scFv-miniSOG is excited by blue light, its application in vivo might require light delivery system coupled with fiber-optic technology allowed the interstitial light delivery [35]. And we hope that well-coordinated interdisciplinary efforts would cope with this challenge. Correct approach might enable in vivo implementation of $4 \mathrm{D} 5 \mathrm{scFv}$-miniSOG cytotoxic potential and could make photodynamic properties of our immunoPS comparable to other commercially available red PSs.

\section{Supplementary Material}

Figure S1. Confocal fluorescence images of SK-BR-3 (A) and $\mathrm{CHO}(\mathrm{B})$ after $1 \mathrm{~h}$ incubation with free miniSOG $(500 \mathrm{nM})$ at $37^{\circ} \mathrm{C}$. Left row, a bright-field image; right row, green fluorescence image.

Figure S2. Relative cell viability of SK-BR-3 cells after treatment with various Taxol concentrations.

http://www.thno.org/v03p0831s1.pdf

\section{Acknowledgments}

We are grateful to Victor B. Loschenov for providing the opportunity to carry out fluorescence measurements. We thank Andre Lieber for plasmid pQE30-K-Ad3-6th-SK.

\section{Grant Support}

This work was supported by the Russian Foundation for Basic Research (projects nos. 12-04-01083-a, 12-04-00757-a, 13-04-40226), by Russian Academy of Science Presidium Programs (Fundamental Sciences for Medicine-2013, Molecular \& Cellular Biology and Nanotechnologies \& Nanomaterials) and by The Ministry of Education and Science of Russian Federation (projects nos. 8279, 16.523.12.3009, 11.G34.31. 0017).

\section{Author contributions}

K.M., G.P., A.R., O.S., S.L., R.P. and S.D. designed research; K.M., G.P., A.R. and O.S. performed research; K.M., G.P. and A.R. analyzed data; G.P., 
K.M. and S.D. wrote the paper.

\section{Competing Interests}

The authors have declared that no competing interest exists.

\section{References}

1. Agostinis P, Berg K, Cengel KA, Foster TH, Girotti AW, Gollnick SO, et al. Photodynamic therapy of cancer: an update. CA Cancer J Clin. 2011; 61: 250-81.

2. Yoo JO, Ha KS. New insights into the mechanisms for photodynamic therapy-induced cancer cell death. Int Rev Cell Mol Biol. 2012; 295: 139-74.

3. Solban N, Rizvi I, Hasan T. Targeted photodynamic therapy. Lasers Surg Med. 2006; 38: 522-31.

4. Verma S, Watt GM, Mai Z, Hasan T. Strategies for enhanced photodynamic therapy effects. Photochem Photobiol. 2007; 83: 996-1005.

5. Mew D, Wat CK, Towers GH, Levy JG. Photoimmunotherapy: treatment of animal tumors with tumor-specific monoclonal antibody-hematoporphyrin conjugates. J Immunol. 1983; 130: 1473-7.

6. van Dongen GA, Visser GW, Vrouenraets MB. Photosensitizer-antibody conjugates for detection and therapy of cancer. Adv Drug Deliv Rev. 2004; 56: 31-52.

7. Staneloudi C, Smith KA, Hudson R, Malatesti N, Savoie H, Boyle RW, et al. Development and characterization of novel photosensitizer: $\mathrm{scFv}$ conjugates for use in photodynamic therapy of cancer. Immunology. 2007; 120: 512-7.

8. Hasan T. Photosensitizer delivery mediated by macromolecular carrier systems. In: Henderson BW and Dougherty TJ, eds. Photodynamic therapy: basic principles and clinical applications. New York: Marcel Dekker; 1992: 187-200.

9. Serebrovskaya EO, Edelweiss EF, Stremovskiy OA, Lukyanov KA, Chudakov DM, Deyev SM. Targeting cancer cells by using an antireceptor antibody-photosensitizer fusion protein. Proc Natl Acad Sci USA. 2009; 106: 9221-5.

10. Brand FX, Ravanel N, Gauchez AS, Pasquier D, Payan R, Fagret D, et al. Prospect for anti-HER2 receptor therapy in breast cancer. Anticancer Res. 2006; 26: 463-70

11. Balandin TG, Edelweiss E, Andronova NV, Treshalina EM, Sapozhnikov AM, Deyev SM. Antitumor activity and toxicity of anti-HER2 immunoRNase $\mathrm{scF} v$ 4D5-dibarnase in mice bearing human breast cancer xenografts. Invest New Drugs. 2011; 29: 22-32.

12. Drebin JA, Link VC, Weinberg RA, Greene MI. Inhibition of tumor growth by a monoclonal antibody reactive with an oncogene-encoded tumor antigen. Proc Natl Acad Sci USA. 1986; 83: 9129-33.

13. Deyev SM, Lebedenko EN. Multivalency: the hallmark of antibodies used for optimization of tumor targeting by design. Bioessays. 2008; 30: 904-18.

14. Shu X, Lev-Ram V, Deerinck TJ, Qi Y, Ramko EB, Davidson MW, et al. A genetically encoded tag for correlated light and electron microscopy of intact cells, tissues, and organisms. PLoS Biol. 2011; 9: e1001041.

15. Qi YB, Garren EJ, Shu X, Tsien RY, Jin Y. Photo-inducible cell ablation in Caenorhabditis elegans using the genetically encoded singlet oxygen generating protein miniSOG. Proc Natl Acad Sci USA. 2012; 109: 7499-504

16. Lin JY, Sann SB, Zhou K, Nabavi S, Proulx CD, Malinow R, et al. Optogenetic Inhibition of Synaptic Release with Chromophore-Assisted Light Inactivation (CALI). Neuron. 2013; 79: 241-53.

17. Ryumina AP, Serebrovskaya EO, Shirmanova MV, Snopova LB, Kuznetsova MM, Turchin IV, et al. Flavoprotein miniSOG as a genetically encoded photosensitizer for cancer cells. Biochim Biophys Acta. 2013; 1830: 5059-67.

18. Bass $\mathrm{S}, \mathrm{Gu} \mathrm{Q}, \mathrm{Christen} \mathrm{A}$. Multicopy suppressors of prc mutant Escherichia coli include two HtrA (DegP) protease homologs (HhoAB), DksA, and a truncated R1pA. J Bacteriol. 1996; 178: 1154-61.

19. Rosenfeld J, Capdevielle J, Guillemot JC, Ferrara P. In-gel digestion of proteins for internal sequence analysis after one- or two-dimensional gel electrophoresis. Anal Biochem. 1992; 203: 173-9.

20. Iselt $M$, Holtei $W$, Hilgard $P$. The tetrazolium dye assay for rapid in vitro assessment of cytotoxicity. Arzneimittelforschung. 1989; 39: 747-9.

21. Deyev SM, Waibel R, Lebedenko EN, Schubiger AP, Plückthun A. Design of multivalent complexes using the barnase*barstar module. Nat Biotechnol. 2003; 21: 1486-92.
22. Eigenbrot C, Randal M, Presta L, Carter P, Kossiakoff AA. X-ray structures of the antigen-binding domains from three variants of humanized anti-p185HER2 antibody 4D5 and comparison with molecular modeling. J Mol Biol. 1993; 229: 969-95.

23. Arnold K, Bordoli L, Kopp J, Schwede T. The SWISS-MODEL workspace: a web-based environment for protein structure homology modelling. Bioinformatics. 2006; 22: 195-201.

24. Schwede T, Kopp J, Guex N, Peitsch MC. SWISS-MODEL: an automated protein homology-modeling server. Nucleic Acids Res. 2003; 31: 3381-5.

25. Wörn A, Plückthun A. An intrinsically stable antibody scFv fragment can tolerate the loss of both disulfide bonds and fold correctly. FEBS Lett. 1998; 427: 357-61.

26. Sarup JC, Johnson RM, King KL, Fendly BM, Lipari MT, Napier MA, et al. Characterization of an anti-p185HER2 monoclonal antibody that stimulates receptor function and inhibits tumor cell growth. Growth Regul. 1991; 1: 72-82.

27. Wang H, Li Z, Yumul R, Lara S, Hemminki A, Fender P, Lieber A. Multimerization of adenovirus serotype 3 fiber knob domains is required for efficient binding of virus to desmoglein 2 and subsequent opening of epithelial junctions. J Virol. 2011; 85: 6390-402.

28. Chen X, Yeung TK, Wang Z. Enhanced drug resistance in cells coexpressing ErbB2 with EGF receptor or ErbB3. Biochem Biophys Res Commun. 2000; 277: 757-63.

29. Beyer I, van Rensburg R, Strauss R, Li Z, Wang H, Persson J, et al. Epithelial junction opener JO-1 improves monoclonal antibody therapy of cancer. Cancer Res. 2011; 71: 7080-90.

30. Bhatti M, Yahioglu G, Milgrom LR, Garcia-Maya M, Chester KA, Deonarain MP. Targeted photodynamic therapy with multiply-loaded recombinant antibody fragments. Int J Cancer. 2008; 122: 1155-63.

31. Savellano MD, Pogue BW, Hoopes PJ, Vitetta ES, Paulsen KD. Multiepitope HER2 targeting enhances photoimmunotherapy of HER2-overexpressing cancer cells with pyropheophorbide-a immunoconjugates. Cancer Res. 2005; 65: 6371-9.

32. Ruiz-González R, Cortajarena AL, Mejias SH, Agut M, Nonell S, Flors C. Singlet Oxygen Generation by the Genetically Encoded Tag miniSOG. J Am Chem Soc. 2013; 135: 9564-7.

33. Pimenta FM, Jensen RL, Breitenbach T, Etzerodt M, Ogilby PR. Oxygen-Dependent Photochemistry and Photophysics of "MiniSOG," a Protein-Encased Flavin. Photochem Photobiol. 2013; 89:1116-26.

34. Firczuk M, Winiarska M, Szokalska A, Jodlowska M, Swiech M, Bojarczuk K, et al. Approaches to improve photodynamic therapy of cancer. Front Biosci. 2011; 16: 208-24.

35. Wilson BC, Patterson MS. The physics, biophysics and technology of photodynamic therapy. Phys Med Biol. 2008; 53: R61-109. 